\title{
STANDARD THERMAL ENERGY GROUP STRUCTURE FOR GENERATION OF THERMAL GROUP CONSTANTS FROM ENDF/B DATA
}

\author{
D. R. FINCH
}

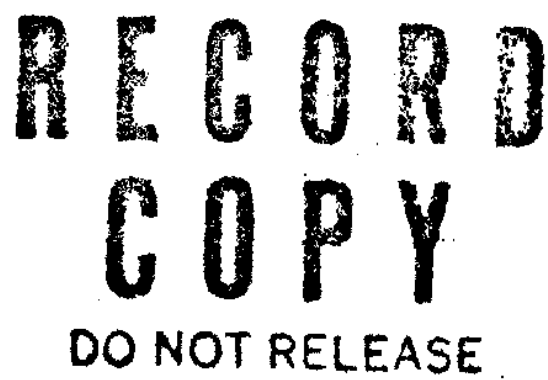

FROM FILE

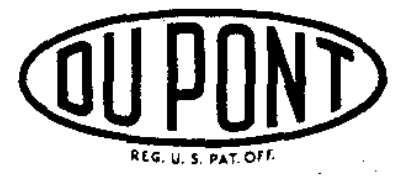

E. I. du Pont de Nemours \& Co.

Savannah River Laboratory

Aiken, S. C. 29801 


\section{NOTICE}

This report was prepared as an account of work sponsored by the United States Government. Neither the United States nor the United States Atomic Energy Commission, nor any of their employees, nor any of their contractors, subcontractors, or their employees, makes any warranty, express or implied, or assumes any legal liability or responsibility for the accuracy, completeness or usefulness of any information, apparatus, product or process disclosed, or represents that its use would not infringe privately owned rights.

Printed in the United States of America Available from

National Technical Information Service

U. S. Department of Commerce

5285 Port Royal Road

Springfield, Virginia 22151

Price: Printed Copy $\$ 4.00$; Microfiche $\$ 1.45$ 
DP-1346

\title{
STANDARD THERMAL ENERGY GROUP STRUCTURE FOR GENERATION OF THERMAL GROUP CONSTANTS FROM ENDF/B DATA
}

\author{
by
}

D. R. Finch

Approved by

J. R. Hilley, Research Manager

Computer Applications Division

Publication Date: March 1974

E. 1. du Pont de Nemours \& Co. Savannah River Laboratory

Aiken, S. C. 29801

PREPARED FOR THE U.S. ATOMIC ENERGY COMMISSION UNDER CONTRACT ATI07-2)-1 


\begin{abstract}
This report contains the final specifications of a standard energy group structure for the generation of thermal group constants from ENDF/B data. The report represents the work of a committee appointed by the Codes and Formats Subcommittee of the Cross Section Evaluation Working Group and is a parallel effort to work being done in the epithermal energy range.

The thermal energy group structure specified in this report was accepted November 10, 1972, by the Cross Section Evaluation Working Group as the standard for generation of thermal group constants from ENDF/B data.

The standard thermal group structure specified in this report is consistent with past design experience and thermal spectrum codes, and incorporates specific features for effects known to be important in nuclear design applications in the thermal energy range.

Specific recommendations are made as to methods to be used for generation of thermal group constants.
\end{abstract}




\section{CONTENTS}

Page

Introduction . . . . . . . . . . . . . . . . . . 5

Specification of the Standard Thermal Energy Group

Structure . . . . . . . . . . . . . . . . . . 7

Energy Group Structure for Smooth Cross Sections . . . . 7

Energy Group Structure for Thermal Scattering

Matrices... . . . . . . . . . . . . . . . . 18

Recommendations for Generation of Thermal Group

Constants . . . . . . . . . . . . . . . . . . . 20

Spectrum . . . . . . . . . . . . . . . . . 20

Resonance Cross Sections . . . . . . . . . . . 20

Scattering Matrices ............... 20

Output Format . . . . . . . . . . . . . . 21

Computer Codes for Generation of Thermal Group

Constants . . . . . . . . . . . . . . . . . . 22

Acknowledgments . . . . . . . . . . . . . . . . . . . 31

References . . . . . . . . . . . . . . . 32 


\section{LIST OF TABLES}

Table

$\underline{\text { Page }}$

I 460 Energy Group Structure for Smooth Cross Sections . . 9

II 50 Energy Group Structure for Thermal Scattering Matrices . . . . . . . . . . . . . . . . 19

III 30 Group THERMOS Scattering Matrices Included in ACC Abstract 543. . . . . . . . . . . . . . . 21

IV 50 Group Thermal Scattering Matrices Included in ACC Abstract 543 . . . . . . . . . . . . . . . . 21

$V$ Thermal Region Cross Section Codes Interfaced to ENDF/B Data . . . . . . . . . . . . . . . 22

VI Equivalences Between Group Structures . . . . . . . . . 23 


\section{INTRODUCTION}

In May 1972, the Chairman of the Codes and Formats Subcommittee of the Cross Section Evaluation Working Group (CSEWG) appointed a committee to:

- Prepare a standard energy group structure spanning the thermal energy region for generating standard ENDF/B thermal group constants

- Recommend procedures for generating thermal group constants from ENDF/B data on this standard group structure

- Recommend an output format for thermal group constants on this standard group structure

The efforts of this special committee were a parallel effort to a similar committee appointed to make recommendations for the fast (epithermal) energy region.

A preliminary report of the thermal region committee was submitted and accepted by the CSEWG Codes and Formats Subcommittee on November 9, 1972. This same report was forwarded to and accepted by the Cross Section Evaluation Working Group on November $10,1972$.

After the epithermal standard group structure was accepted by CSEWG, very sma11 modifications were necessary to resolve discrepancies in the interface region between the epithermal and thermal group structures.

This report represents a final set of specifications of the standard thermal energy group structure accepted by the Cross Section Evaluation Working Group for generation of thermal group constants from ENDF/B data. 


\section{SPECIFICATION OF THE THERMAL ENERGY GROUP STRUCTURE}

\section{ENERGY GROUP STRUCTURE FOR SMOOTH CROSS SECTIONS}

In setting out the specifications of a thermal energy group structure for smooth thermal cross sections, three questions were asked.

1. How high in energy should the group structure extend?

Different thermal spectra reactors require a different high energy cutoff. What is desired is to select a high enough cutoff energy so that all thermal spectra reactor needs are satisfied.

2. What energy group breaks are necessary to enable interface to standard thermal spectrum codes?

A vast amount of money and time has been spent on developing the existing therma1 spectrum codes. Any standard group structure should make every effort to interface as many of these as possible.

3. Are the energy groups sufficiently narrow to enable a unit weighting spectrm to be used in averaging the group constants?

Because the group structure is to be valid for all thermal spectra, it is necessary to produce group constants from ENDF/B using a unit spectrum to preserve the integrity of the ENDF/B data.

The first question was resolved by discussing it with several persons involved in design and/or analysis of thermal reactor lattices. The upper energy was chosen as $3.15 \mathrm{eV}$ to provide energy groups significantly higher in energy than the effects of the $1.056 \mathrm{eV}$ resonance in $240 \mathrm{Pu}$, and simultaneously to span the $3.059 \mathrm{eV}$ group break of the MUFT ${ }^{9}$ and GAM $^{10}$ slowing-down codes.

The second question was resolved by compiling a compendium of group breaks for the commonly used thermal spectrum codes. The specific codes included in this compendium were the following: 


$\begin{array}{ll}\text { THERMOS }^{2} & 30 \text { groups } \\ \text { LASER }^{3} & 35 \text { groups } \\ \text { KATE }^{4} & 246 \text { groups } \\ \text { GATHER II }^{5} & 101 \text { groups } \\ \text { GATHER (Rev.) }^{5} & 101 \text { groups } \\ \text { TEMPEST }^{6} & 246 \text { and } 309 \text { groups } \\ \text { SOFOCATE }^{7} & 246 \text { and } 309 \text { groups } \\ \text { LEOPARD }^{8} & 172 \text { and } 309 \text { groups } \\ \text { MUFT III, IV, V }^{9} & \text { Group breaks }<3.15 \mathrm{eV} \\ \text { GAM I and II } & \text { Group breaks }<3.15 \mathrm{eV}\end{array}$

The result of this compendium was a group structure comprising 482 groups and a high energy cutoff of $3.15 \mathrm{eV}$. Many of the group breaks in this structure were very close together $(<1 / 4 \%)$; hence, slight shifts of group boundaries were made to eliminate those breaks that yielded physically insignificant energy groups.

No shifts were introduced that could conceivabiy alter group constants, and care was taken to preserve the MUFT and GAM group breaks where they overlap the thermal energy group structure.

An examination of the resulting energy group structure containing 460 energy groups showed that Question 3 was easily satisfied by this structure.

The 460 energy group structure shown in Table $I$ is the standard group structure for generation of thermal group constants from ENDF/B data.

Table I contains all energy breaks for the 460 group set expressed to five significant digits. Also shown is the group structure(s) from which the particular energy break arose. The spectrum codes are designated by a code number in the table, and the key is given below.

\begin{tabular}{cl} 
Code Number & Group Structure Name \\
\cline { 3 - 3 } 2 & MUFT II I , IV, V \\
3 & GAM I and II \\
4 & THERMOS - 30 groups \\
5 & LASER - 35 groups \\
6 & H20 KERNEL - 50 groups \\
7 & GATHER II \\
8 & GATHER (REV) \\
9 & TEMPEST - 246 groups \\
& TEMPEST - 309 groups
\end{tabular}


TABLE I

460 Energy Group Structure for Smooth Cross Sections

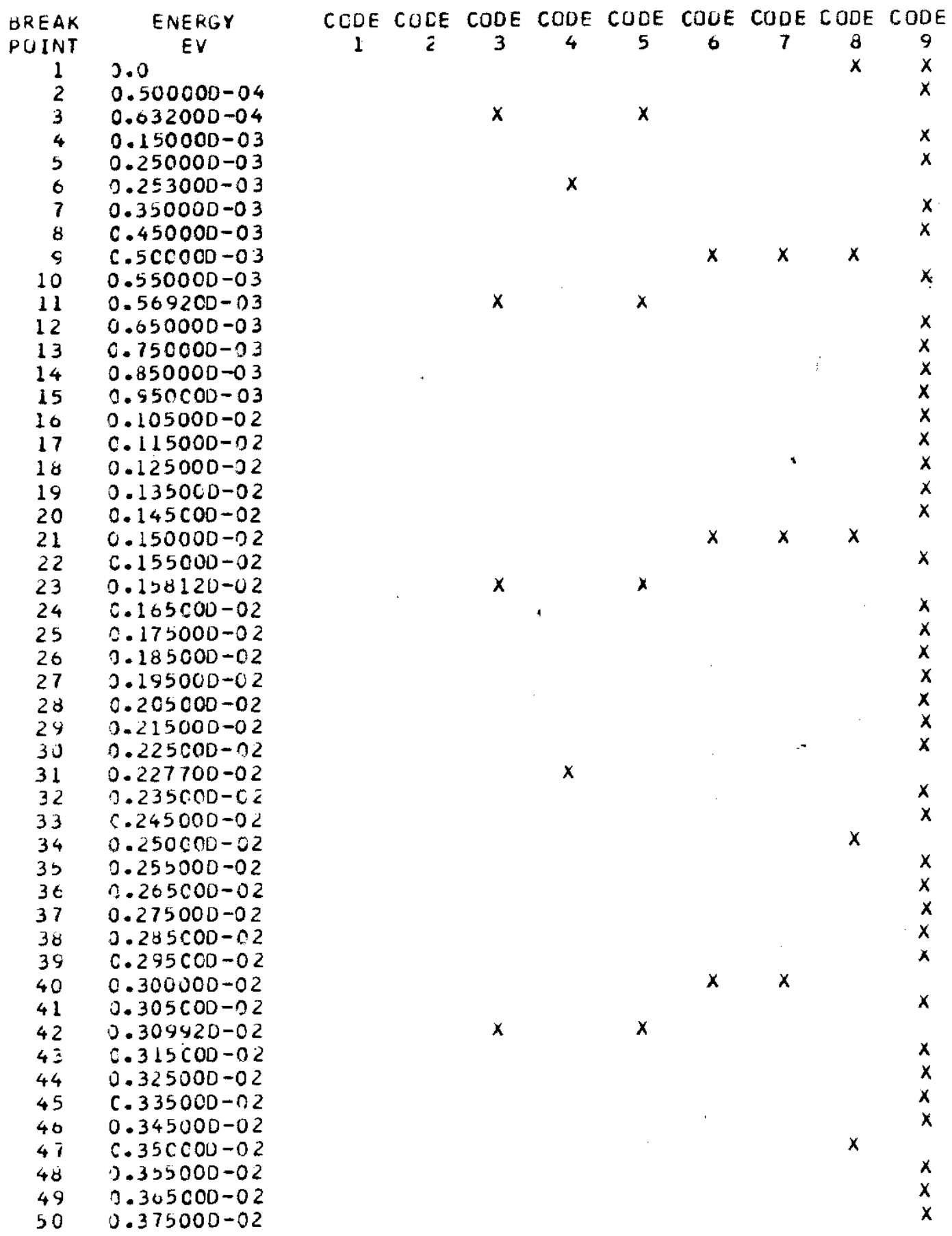




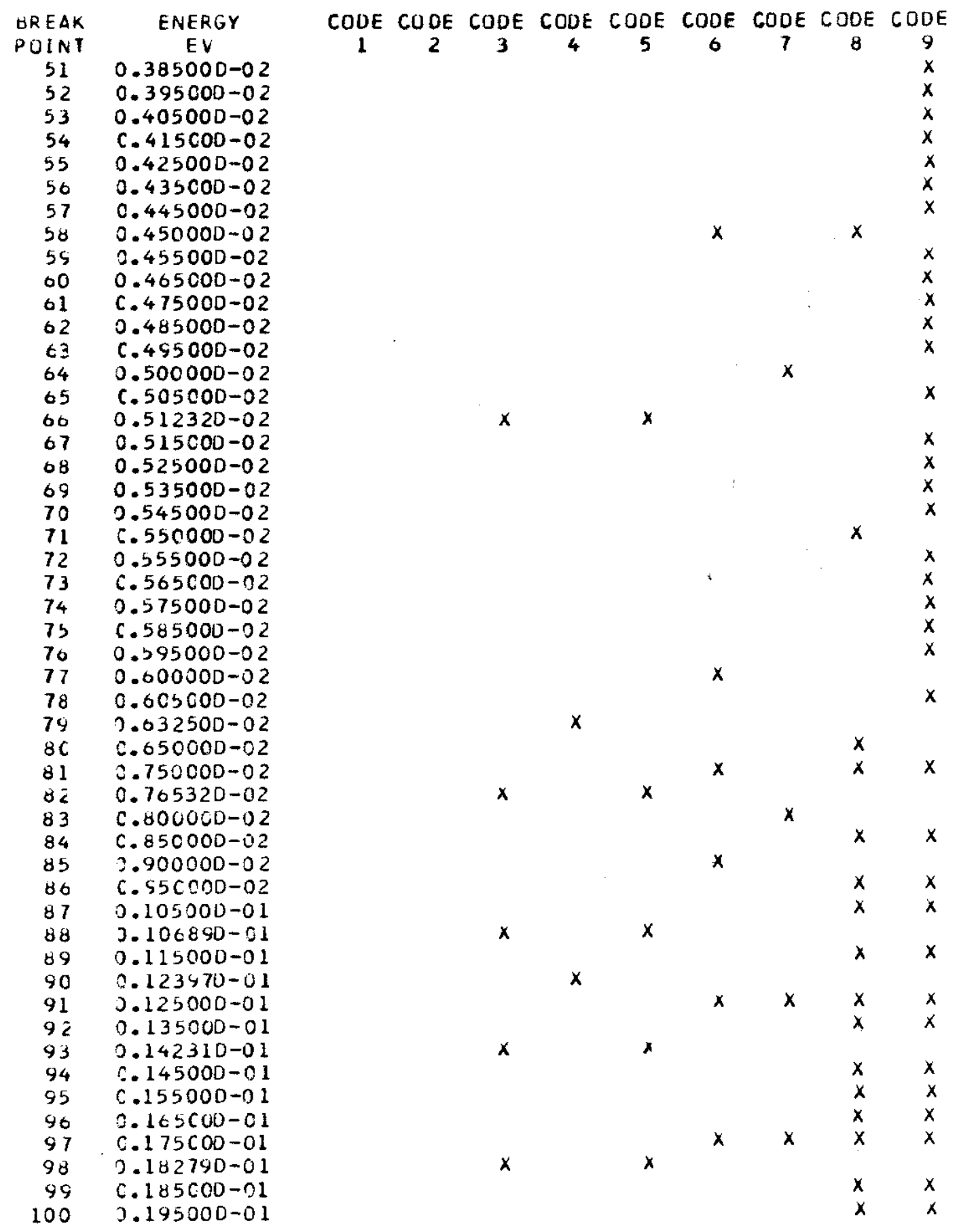




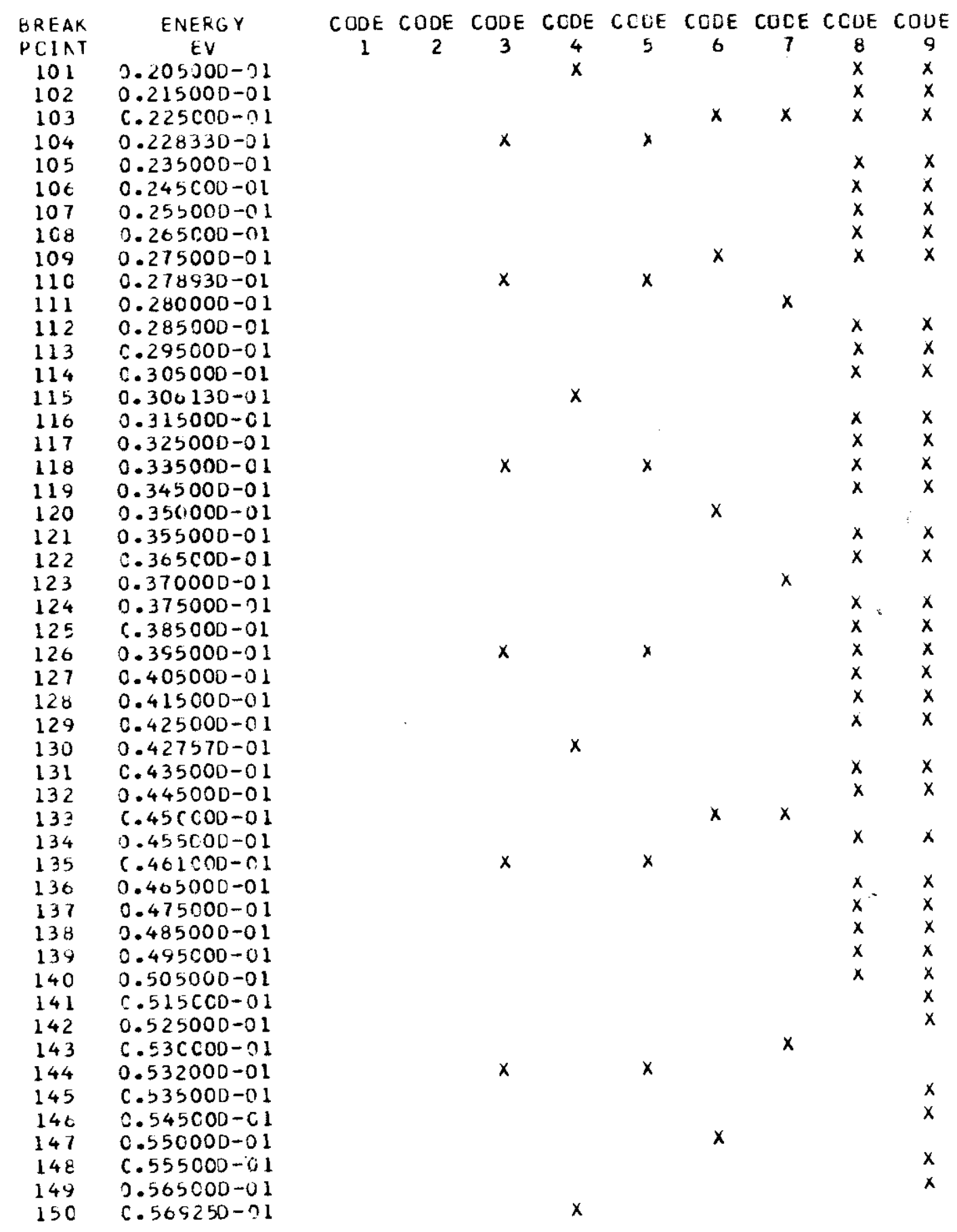




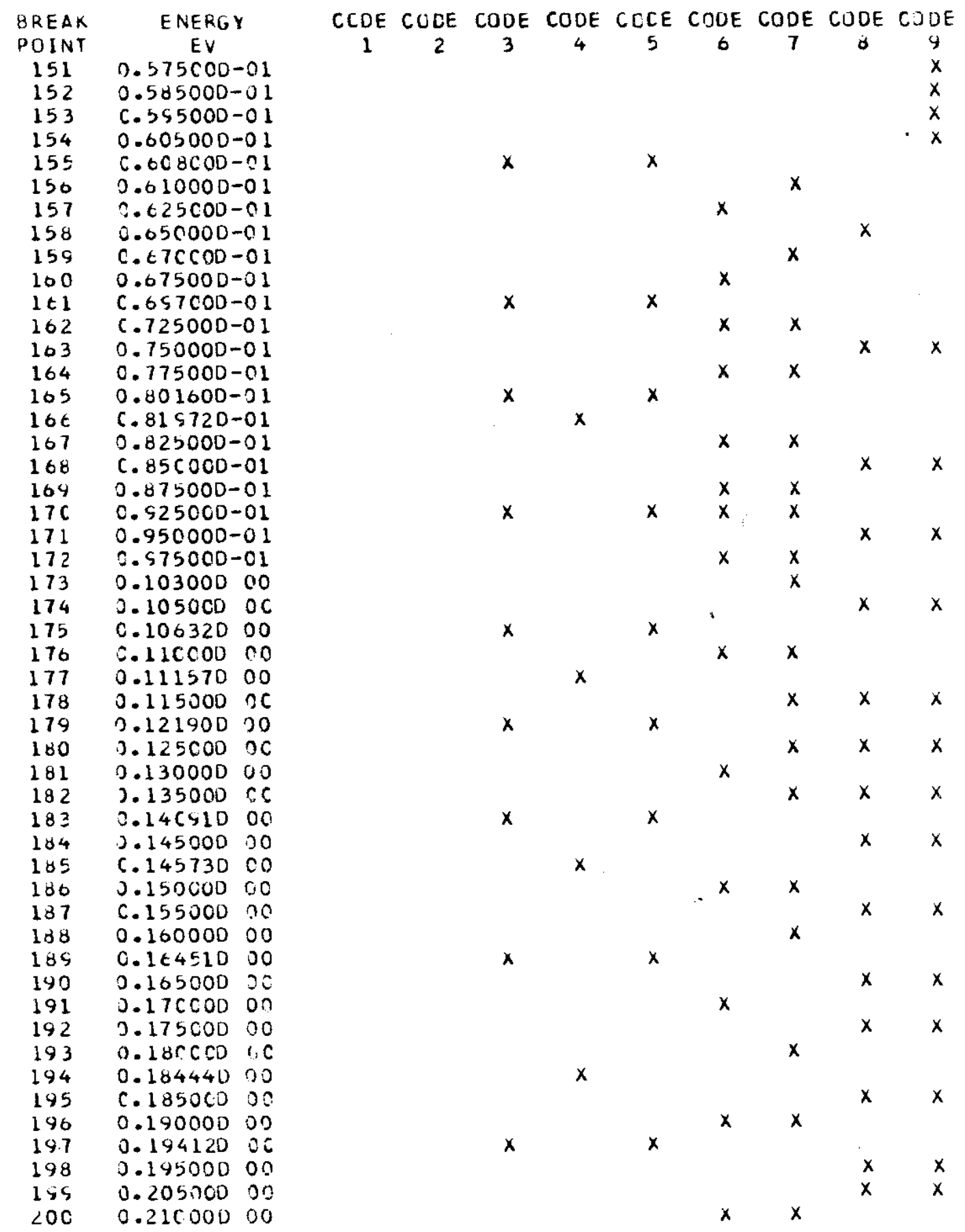




\begin{tabular}{|c|c|c|}
\hline $\begin{array}{l}\text { GREAK } \\
\text { PUINT }\end{array}$ & $\begin{array}{c}\text { ENERG } \\
\text { EV }\end{array}$ & \\
\hline 201 & 0.215000 & 00 \\
\hline 202 & $0.2200 \mathrm{co}$ & $O \mathrm{C}$ \\
\hline 203 & $C .225000$ & 00 \\
\hline 204 & 227700 & 0 \\
\hline 205 & 231500 & 0 \\
\hline 206 & 235000 & 9 \\
\hline 207 & 0.245000 & 0 \\
\hline 208 & 0.25 & \\
\hline 209 & 000 & \\
\hline 210 & 00 & \\
\hline 211 & 30 & \\
\hline 212 & 0.275000 & \\
\hline 213 & 278870 & 0 \\
\hline 214 & 285000 & 0 \\
\hline 215 & 750 & 0 \\
\hline 210 & $O D$ & 0 \\
\hline 217 & 01130 & \\
\hline 218 & 305000 & 0 \\
\hline 219 & $315 \mathrm{COD}$ & 0 \\
\hline 220 & $20 \in 40$ & 0 \\
\hline 221 & $325 \mathrm{COD}$ & 0 \\
\hline 222 & 335000 & 0 \\
\hline 223 & 338910 & 0 \\
\hline 224 & 345000 & 0 \\
\hline 225 & $355 \mathrm{COD}$ & 0 \\
\hline 220 & 357080 & 0 \\
\hline 227 & 65000 & 0 \\
\hline 228 & 00 & 0 \\
\hline 229 & $0 D$ & 0 \\
\hline 230 & 100 & 0 \\
\hline 231 & 390000 & 0 \\
\hline 232 & 5000 & \\
\hline 233 & 405000 & 0 \\
\hline 234 & 990 & 0 \\
\hline 235 & 000 & 0 \\
\hline 236 & $C 4 D$ & 0 \\
\hline 237 & 000 & 0 \\
\hline 238 & 00 & 0 \\
\hline 239 & 000 & 0 \\
\hline $24 C$ & 500 & 0 \\
\hline 241 & 000 & 0 \\
\hline 242 & 455000 & 0 \\
\hline 243 & 00000 & 0 \\
\hline 244 & 465000 & $c$ \\
\hline 245 & 0.472500 & 0 \\
\hline $24 t$ & .475000 & 0 \\
\hline 247 & 0.477500 & 0 \\
\hline 248 & 485000 & 0 \\
\hline 249 & 90000 & 0 \\
\hline 260 & 000 & \\
\hline
\end{tabular}

CODE CODE COOE CODE CUDE CODE CODE CODE CODE

$\begin{array}{lllllllll}1 & 2 & 3 & 4 & 5 & 6 & 7 & 8 & 9 \\ & & & & & & & x & x\end{array}$

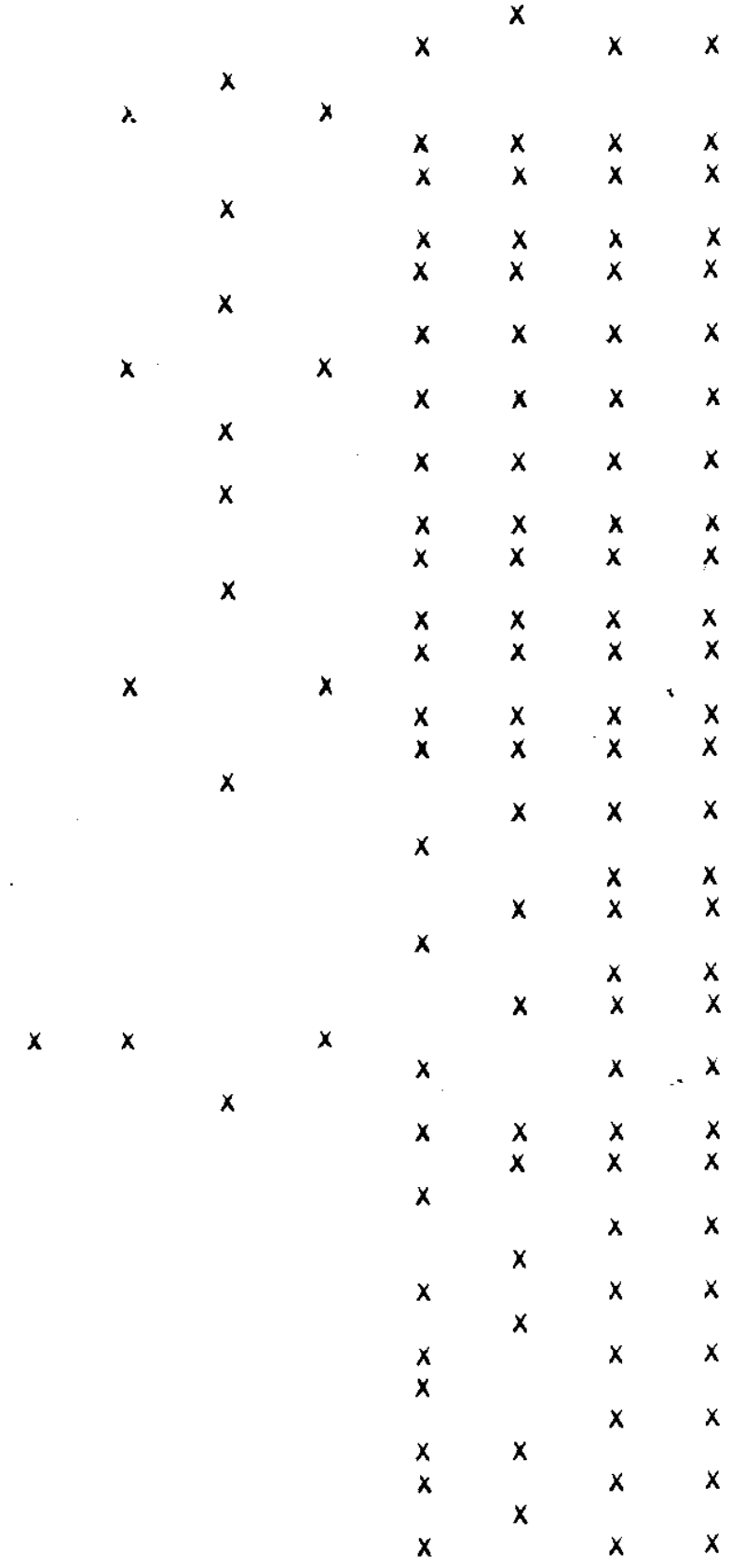




\begin{tabular}{|c|c|c|c|c|c|c|c|c|c|c|c|}
\hline $\begin{array}{l}\text { SREAK } \\
\text { PCINT }\end{array}$ & ENERG & & CODE & CODE & CODE & CCDE & CCDE & CCDE & CODE & CODE & $\underset{9}{C O O E}$ \\
\hline PCINT & EV & & & & & 4 & & & & & \\
\hline$<51$ & $C .503260$ & 00 & & & & $x$ & & & & & \\
\hline $\begin{array}{l}252 \\
753\end{array}$ & 0.505000 & 00 & & & & & & & $x$ & $x$ & $x$ \\
\hline 253 & C. 511180 & 00 & & & $x$ & & $x$ & & & & \\
\hline 234 & 0.515000 & 00 & & & & & & $x$ & & $x$ & $x$ \\
\hline 255 & $0.52 \mathrm{C000}$ & 00 & & & & & & & $x$ & & \\
\hline 250 & 0.525000 & 00 & & & & & & & & $x$ & $x$ \\
\hline 257 & 0.531580 & $O C$ & & $x$ & & & & & & & \\
\hline 258 & 0.535000 & 00 & & & & & & & & $x$ & $x$ \\
\hline 259 & 0.540000 & 00 & & & & & & $x$ & & & \\
\hline 260 & $0.545 C 00$ & $\mathrm{CC}$ & & & & & & & $x$ & $x$ & $x$ \\
\hline 261 & 0.555000 & 00 & & & & & & & & $x$ & $x$ \\
\hline $2 \in 2$ & 0.560000 & 00 & & & & & & $x$ & & & \\
\hline 263 & 6.565000 & 00 & & & & & & & $x$ & $x$ & $x$ \\
\hline 264 & $C .575000$ & $\mathrm{CC}$ & & & & & & & & $x$ & $x$ \\
\hline 265 & 0.580000 & 00 & & & & & & $x$ & & & \\
\hline $2 \in t$ & C. 585000 & 00 & & & & & & & $x$ & $x$ & $x$ \\
\hline 267 & 0.595000 & 00 & & & & & & $x$ & & $x$ & $x$ \\
\hline 268 & 0.605000 & 00 & & & & & & & & $x$ & $x$ \\
\hline 269 & 0.615000 & 00 & & & & & & $x$ & $x$ & $x$ & $x$ \\
\hline $27 \mathrm{C}$ & 0.625060 & 00 & $x$ & & & $x$ & & & & $x$ & $x$ \\
\hline 271 & 0.632500 & 00 & & & $x$ & & $x$ & & & & \\
\hline 272 & $0.63500 D$ & 00 & & & & & & $x$ & $x$ & $x$ & $x$ \\
\hline 273 & $0.645 \mathrm{COD}$ & 00 & & & & & & & 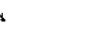 & $x$ & $x$ \\
\hline 274 & 0.655000 & 00 & & & & & & & & $x$ & $x$ \\
\hline 275 & 0.665000 & 00 & & & & & & & $x$ & $x$ & $x$ \\
\hline 276 & 0.670000 & 00 & & & & & & $x$ & & & \\
\hline 277 & C. $675 \mathrm{COD}$ & 00 & & & & & & & & $x$ & $x$ \\
\hline 278 & $0.68256 \mathrm{D}$ & 00 & & $x$ & & & & & & & \\
\hline 279 & 0.685000 & $O C$ & & & & & & & $x$ & $\mathbf{x}$ & $x$ \\
\hline 280 & 0.090000 & 00 & & & & & & $x$ & & & \\
\hline 281 & 0.655000 & 00 & & & & & & & & $x$ & $x$ \\
\hline 282 & 0.705000 & 00 & & & & & & & & $x$ & $x$ \\
\hline 283 & C.715COD & CC & & & & & & & $x$ & $x$ & $x$ \\
\hline 284 & 0.725000 & DO & & & & & & $x$ & & $x$ & $x$ \\
\hline 285 & C. 735000 & 00 & & & & & & & $=$ & $x$ & $x$ \\
\hline 280 & 0.745000 & 00 & & & & & & & & $x$ & $x$ \\
\hline 287 & 0.755000 & OC & & & & & & & $x$ & $x$ & $x$ \\
\hline 288 & 0.705000 & 00 & & & & & & & & $x$ & $x$ \\
\hline 289 & 0.775000 & 00 & & & & & & $x$ & & $x$ & $x$ \\
\hline $29 \mathrm{C}$ & 0.782110 & 00 & & & & $x$ & & & & & \\
\hline 291 & 0.785000 & 50 & & & $x$ & & $x$ & & & $x$ & $x$ \\
\hline 292 & c. 755000 & 00 & & & & & & & & $x$ & $x$ \\
\hline 293 & 6.805000 & 00 & & & & & & & $x$ & $x$ & $x$ \\
\hline 294 & C. 815000 & 00 & & & & & & & & $x$ & $x$ \\
\hline 295 & 0.825000 & 00 & & & & & & $x$ & & $x$ & $x$ \\
\hline $29 t$ & C. 833680 & 00 & $x$ & & & & $x$ & & & $x$ & $x$ \\
\hline 297 & 0.840000 & co & & & & & & & $x$ & . & \\
\hline 298 & 0.845000 & 00 & & & & & & & & $x$ & $x$ \\
\hline 299 & 0.855000 & 00 & & & & & & & & $x$ & $x$ \\
\hline $30 \mathrm{c}$ & C. 860000 & 00 & & & & & & $x$ & & & \\
\hline
\end{tabular}




\begin{tabular}{|c|c|c|c|c|c|c|c|c|c|c|c|}
\hline BREAK & ENERGY & & CCDE & COCE & CODE & CCOE & CCCE & CODE & CODE & COUE & COUE \\
\hline POINT & EV & & 1 & 2 & 3 & 4 & 5 & 6 & 7 & 8 & 9 \\
\hline 301 & C. .665000 & co & & & & & & & & & \\
\hline 302 & 0.870000 & 00 & & & & & & & $x$ & & \\
\hline $3 C_{3}$ & C.8.7642D & 00 & & $x$ & & & $x$ & & & $x$ & $x$ \\
\hline 304 & $c .380000$ & 00 & & & & & & $x$ & & & \\
\hline 305 & C.\&E5COD & 00 & & & & & & & & $x$ & $x$ \\
\hline 300 & 3.895000 & 00 & & & & & & & & $x$ & $x$ \\
\hline 3107 & $0.900 C 00$ & OC & & & & & & $x$ & & & \\
\hline 308 & 0.905000 & vo & & & & & & & & $x$ & $x$ \\
\hline 309 & 0.910000 & 00 & & & & & $x$ & & $x$ & & \\
\hline 310 & 0.915000 & 00 & & & & & & & & $x$ & $x$ \\
\hline 311 & C. $\$ 200 \mathrm{CD}$ & 0 & & & & & & $x$ & & & \\
\hline 312 & 0.925000 & 00 & & & & & & & & $x$ & $x$ \\
\hline 313 & $\therefore .535000$ & $n 0$ & & & & & & & & $x$ & $x$ \\
\hline 314 & 0.940000 & 00 & & & & & & $x$ & $x$ & & \\
\hline 315 & 0.545000 & 00 & & & & & $x$ & & & $x$ & $x$ \\
\hline 316 & 0.950700 & 00 & & & & $x$ & & & & & \\
\hline 317 & 0.955000 & 00 & & & & & & & & $x$ & $x$ \\
\hline 318 & C. 960000 & 00 & & & & & & $x$ & & & \\
\hline 319 & 0.965000 & 00 & & & & & & & & $x$ & $x$ \\
\hline 320 & C.57C000 & Do & & & & & & & $x$ & & \\
\hline 321 & 0.975000 & 00 & & & & & $x$ & $x$ & & $x$ & $x$ \\
\hline 322 & c. $\$ 85000$ & 00 & & & & & & $x$ & & $x$ & $x$ \\
\hline 323 & 0.955000 & so & & & & & & $x$ & $x$ & $x$ & $x$ \\
\hline 324 & C.1C0500 & 01 & & & & & & & $x$ & $x$ & $x$ \\
\hline 325 & 0.101000 & 01 & & & & & & $x$ & & v & \\
\hline 326 & 0.101500 & 01 & & & & $x$ & $x$ & & & $x$ & $x$ \\
\hline 327 & 0.102500 & 01 & & & & & & & $x$ & $x$ & $x$ \\
\hline 328 & 0.103500 & 01 & & & & & & $x$ & $x$ & $x$ & $x$ \\
\hline 325 & 0.104500 & 01 & & & & $x$ & $x$ & & $x$ & $x$ & $x$ \\
\hline 333 & $\therefore .105500$ & 61 & & & & $x$ & & $x$ & $x$ & $x$ & $x$ \\
\hline 331 & 0.100500 & 01 & & & & $x$ & $x$ & $x$ & $x$ & $x$ & $x$ \\
\hline 332 & $\therefore 107220$ & 01 & & & & $x$ & & & & & \\
\hline 333 & 0.107500 & 01 & & & & & & $x$ & & $x$ & $x$ \\
\hline 334 & $3.108=00$ & $\mathrm{Cl}$ & & & & & $x$ & $x$ & $x$ & $x$ & $x$ \\
\hline 335 & 0.109500 & 01 & & & & & & & $x$ & $x$ & $x$ \\
\hline 336 & 0.110000 & 01 & & & & $x$ & & $x$ & & & \\
\hline 337 & C.11C500 & 01 & & & & & $x$ & & & $=x$ & $x$ \\
\hline 338 & 0.111500 & 01 & & & & & & & $x$ & $x$ & $x$ \\
\hline 339 & 0.112000 & 01 & & & & & & $x$ & $x$ & & \\
\hline 340 & 3.112540 & 01 & $x$ & $x$ & & & $x$ & & & $x$ & $x$ \\
\hline 341 & $c .112750$ & 01 & & & & & & $x$ & & & \\
\hline 342 & 0.113000 & 01 & & & & & & & $x$ & & \\
\hline 343 & 0.113500 & 01 & & & & & & & & $x$ & $x$ \\
\hline 344 & 0.114300 & 01 & & & & & & $x$ & & & \\
\hline 345 & C.114500 & 01 & & & & & & & $x$ & $x$ & $x$ \\
\hline 340 & 0.115500 & 01 & & & & & & & & $x$ & $x$ \\
\hline 347 & 0.116500 & 31 & & & & $x$ & & & & $x$ & $x$ \\
\hline 348 & 0.117500 & 01 & & & & & & $x$ & $x$ & $x$ & $x$ \\
\hline 349 & 0.118500 & 61 & & & & & & & & $x$ & $x$ \\
\hline 350 & 0.119500 & 01 & & & & & & & & $x$ & $x$ \\
\hline
\end{tabular}




\begin{tabular}{|c|c|c|c|c|c|c|c|c|c|c|c|}
\hline BREAK & ENERG & & CODE & CODE & CODE & CODE & COOE & COUE & CODE & CODE & $\begin{array}{c}\text { CODE } \\
9\end{array}$ \\
\hline POINT & $\begin{array}{c}\text { EV } \\
C 120500\end{array}$ & & & 2 & & 4 & 5 & & 7 & 8 & $\begin{array}{l}9 \\
x\end{array}$ \\
\hline $\begin{array}{l}351 \\
352\end{array}$ & $\begin{array}{l}c .120500 \\
c .121500\end{array}$ & $\begin{array}{l}01 \\
01\end{array}$ & & & & & & & $x$ & $\begin{array}{l}x \\
x\end{array}$ & $\begin{array}{l}x \\
x\end{array}$ \\
\hline 353 & 0.122500 & 01 & & & & & $x$ & $x$ & & $\hat{x}$ & $x$ \\
\hline 354 & C.123500 & 01 & & & & & & & & $x$ & $x$ \\
\hline 355 & 0.124500 & 01 & & & & & & & & $x$ & $x$ \\
\hline 356 & 0.125500 & 01 & & & & & & & & $x$ & $x$ \\
\hline 357 & 0.126500 & 01 & & & & & & & & $x$ & $x$ \\
\hline 358 & 0.127500 & 01 & & & & & & $x$ & $x$ & $x$ & $x$ \\
\hline 359 & 0.128500 & 01 & & & & & & & & $x$ & $x$ \\
\hline 360 & 0.129500 & 01 & & & & & & & & $x$ & n \\
\hline 361 & 0.130500 & 01 & & & & & & & & $x$ & $x$ \\
\hline 362 & 0.130790 & 01 & & & & $x$ & & & & & \\
\hline 363 & 0.131500 & 01 & & & & & & & & $x$ & \\
\hline 364 & 0.132500 & 01 & & & & & $x$ & $x$ & $x$ & $x$ & \\
\hline 365 & C.133500 & 01 & & & & & & & & $x$ & \\
\hline 366 & 0.134500 & 01 & & & & & & & & $x$ & $x$ \\
\hline 367 & 0.135500 & 01 & & & & & & & & $x$ & \\
\hline 368 & 0.136500 & 01 & & & & & & & & $x$ & $x$ \\
\hline 369 & 0.137500 & 01 & & & & & & & , & $x$ & ג \\
\hline 370 & 0.138500 & 01 & & & & & & & & $x$ & $x$ \\
\hline 371 & 0.135500 & 01 & & & & & & & & $x$ & \\
\hline 372 & 0.140000 & 01 & & & & & & $x$ & $x$ & & \\
\hline 373 & C. $14 C 500$ & 01 & & & & & & & & $x$ & $x$ \\
\hline 374 & 0.141500 & 01 & & & & & . & & & $x$ & 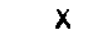 \\
\hline 375 & C. 142500 & 01 & & & & & & & & $x$ & $x$ \\
\hline 376 & 0.143500 & 01 & & & & & & & & $x$ & \\
\hline 377 & 0.143550 & 01 & $\mathrm{x}$ & & & & $x$ & & & & \\
\hline 378 & 0.144500 & 01 & & $x$ & & & & & & $x$ & $x$ \\
\hline 375 & 0.145500 & 01 & & & & & & & & $x$ & \\
\hline 380 & 0.146000 & 01 & & & & $x$ & & & $x$ & & \\
\hline 381 & 0.146500 & $C 1$ & & & & & & & & $x$ & \\
\hline 382 & 0.147000 & 01 & & & & & & $x$ & & & \\
\hline 383 & 0.147500 & $\mathrm{Cl}$ & & & & & & & & $x$ & \\
\hline 384 & 0.148500 & 01 & & & & & & & & $x$ & \\
\hline 385 & 0.149500 & 01 & & & & & & & & $x$ & \\
\hline 386 & 50500 & 01 & & & & & $\therefore$ & & & $x$ & \\
\hline 387 & 0.151500 & 01 & & & & & & & & $x$ & \\
\hline 388 & 0.152500 & C1 & & & & & & & & $x$ & \\
\hline 389 & 0.153500 & 01 & & & & & & & & $x$ & $x$ \\
\hline 390 & C. 154500 & $C 1$ & & & & & & & & $x$ & $\lambda$ \\
\hline 391 & 0.155000 & 01 & & & & & & $x$ & $x$ & & \\
\hline 392 & 5500 & $C 1$ & & & & & & & & $x$ & \\
\hline 393 & 0.156500 & 01 & & & & & & & & $x$ & \\
\hline 394 & 57500 & $n 1$ & & & & & & & & $x$ & \\
\hline 395 & 0.158500 & 01 & & & & & & & & $x$ & \\
\hline $39 t$ & 0.159500 & 01 & & & & $x$ & & & & $x$ & \\
\hline 397 & 160500 & 01 & & & & & & & & $x$ & \\
\hline 398 & 61500 & 01 & & & & & & & & $x$ & \\
\hline 399 & 62500 & 01 & & & & & & & & $x$ & \\
\hline 400 & .163500 & 01 & & & & & $x$ & & & $x$ & \\
\hline
\end{tabular}




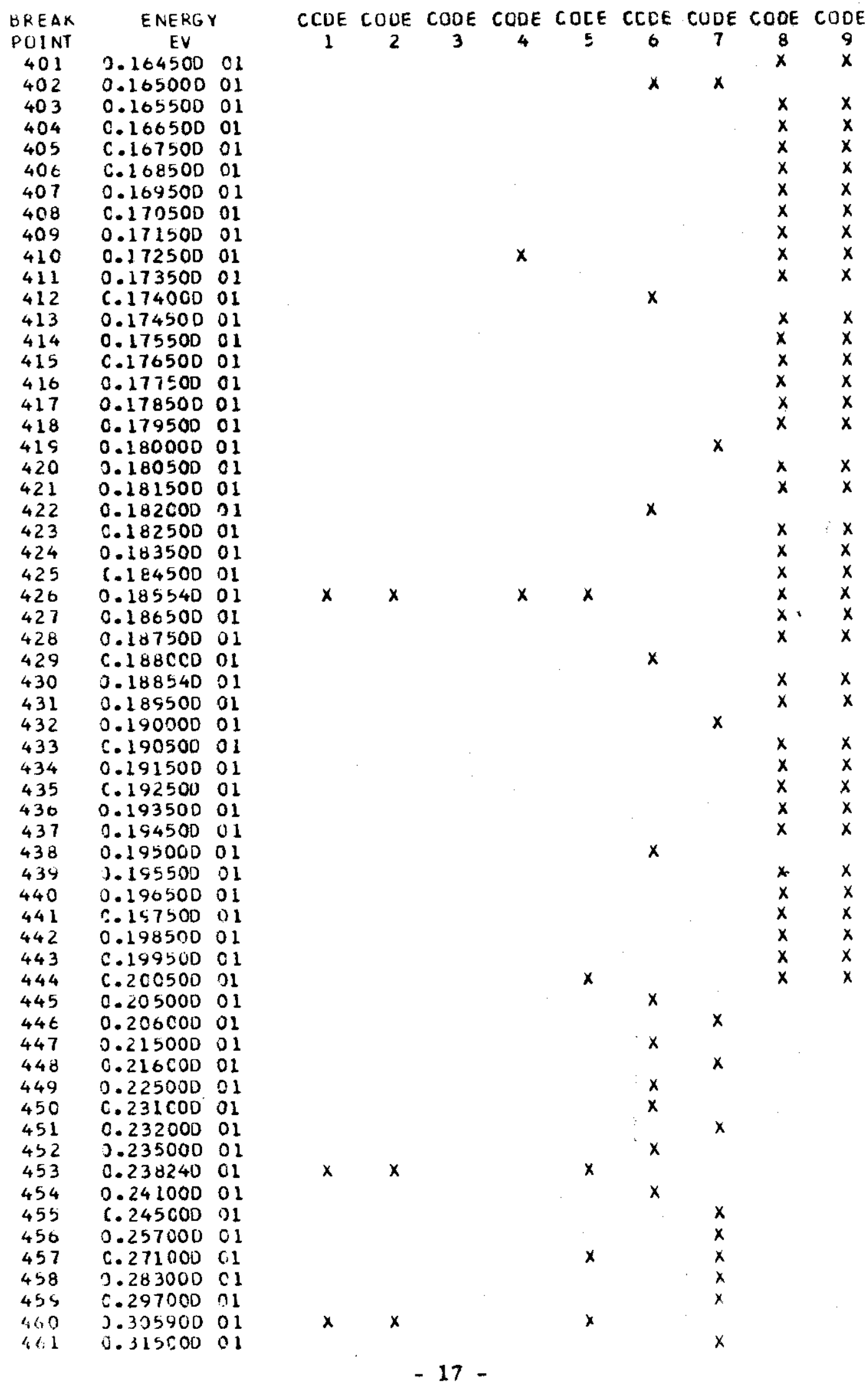




\section{ENERGY GROUP STRUCTURE FOR THERMAL SCATTERING MATRICES}

A necessary part of any set of thermal region group constants are thermal scattering matrices. These matrices contain molecular structure, lattice binding, and upscattering in addition to normal scattering effects, and require considerable computer time to generate from ENDF/B $S(\alpha, \beta)$ data.

It is not within the reasonable limits of existing computers to generate thermal scattering matrices on the 460 energy group structure. Further, matrices generated on such a group structure would be of only limited use. A typical user requires matrices on his specific group structure; hence, matrices must be reduced to his structure by appropriate weighting and averaging. This is possible only if the high and low energies of his group structure are identical to a standard group structure; otherwise, the detailed balance condition of all thermal scattering matrices generated from ENDF/B data will be destroyed. By making arbitrary assumptions, it is possible to restore the detailed balance, but not the physical meaning of the matrices.

Thermal scattering matrices should, therefore," be prepared specifically for a user's energy group structure. Any standardization of thermal scattering matrices can have meaning only for a restricted set of problems and conditions such as thermal data testing.

It is desirable for the purpose of thermal data testing within the CSEWG community to specify a common energy group structure for thermal scattering matrices. The selection of this group structure is, however, based on choosing a group structure of wide usage, familiarity, and general applicability to reactor lattices.

Table II shows a standard 50 energy group structure specified for the purpose of thermal data testing within CSEWG. This energy group structure was obtained from the widely used 30 energy group structure of the THERMOS thermal spectrum code by adding 20 additional energy groups to a high energy cutoff of $3.15 \mathrm{eV}$.

The 50 energy group structure of Table II extends to energies much higher than is typically required in most reactor lattices. In most cases the 30 group THERMOS group structure is adequate (Groups 1-30 of Table II) for most moderator materials. For $\mathrm{H}_{2} \mathrm{O}$-moderated lattices, however, upscattering effects can occur significantly higher in energy than $1.0 \mathrm{eV}$. For lattices with large amounts of $2{ }^{\circ} \mathrm{Pu}$, ignoring the upscattering effects leads to a serious underprediction of the ${ }^{240} \mathrm{Pu}$ reaction rates. The 50 energy group structure of Table II is designed to allow this 
effect and other similar effects to be accounted for in data testing. Where no such effects are present, the more widely known 30 group THERMOS energy group structure is recommended for data testing.

TABLE II

50 Energy Group Structure for Thermal Scattering Matrices

\begin{tabular}{|c|c|c|c|c|c|}
\hline Group & $\begin{array}{l}\text { Avg Energy, } \\
\mathrm{eV}\end{array}$ & Energy Range, $\mathrm{eV}$ & Group & $\begin{array}{c}\text { Avg Energy, } \\
\mathrm{eV}\end{array}$ & Energy Range, eV \\
\hline 1 & 0.000253 & $0.0000632-0.0005692$ & 26 & 0.30815 & $0.27886-0.33891$ \\
\hline 2 & 0.001012 & $0.0005692-0.001581$ & 27 & 0.37551 & $0.33891-0.41399$ \\
\hline 3 & 0.002277 & $0.001581-0.003099$ & 28 & 0.46131 & $0.41399-0.51118$ \\
\hline 4 & 0.004048 & $0.003099-0.005123$ & 29 & 0.57002 & $0.51118-0.63250$ \\
\hline 5 & 0.006325 & $0.005123-0.007653$ & 30 & 0.70668 & $0.63250-0.78500$ \\
\hline 6 & 0.009108 & $0.007653-0.010689$ & 31 & 0.80980 & $0.78500-0.83500$ \\
\hline 7 & 0.012397 & $0.010689-0.014231$ & 32 & 0.85487 & $0.83500-0.87500$ \\
\hline 8 & 0.016192 & $0.014231-0.018279$ & 33 & 0.89240 & $0.87500-0.91000$ \\
\hline 9 & 0.020493 & $0.018279-0.022833$ & 34 & 0.92741 & $0.91000-0.94500$ \\
\hline 10 & 0.025300 & $0.022833-0.027893$ & 35 & 0.95993 & $0.94500-0.97500$ \\
\hline 11 & 0.030632 & $0.027843-0.033500$ & 36 & 0.99489 & $0.97500-1.01500$ \\
\hline 12 & 0.036438 & $0.033500-0.039500$ & 37 & 1.02990 & $1.01500-1.04500$ \\
\hline 13 & 0.042736 & $0.039500-0.046100$ & 38 & 1.05500 & $1.04500-1.06500$ \\
\hline 14 & 0.049586 & $0.046100-0.053200$ & 39 & 1.07500 & $1.06500-1.08500$ \\
\hline 15 & 0.056936 & $0.053200-0.060800$ & 40 & 1.09500 & $1.08500-1.10500$ \\
\hline 16 & 0.065174 & $0.060800-0.069700$ & 41 & 1.11500 & $1.10500-1.12540$ \\
\hline 17 & 0.074838 & $0.069700-0.080160$ & 42 & 1.17450 & $1.12540-1.22500$ \\
\hline 18 & 0.086219 & $0.080160-0.092500$ & 43 & 1.27450 & $1.22500-1.32500$ \\
\hline 19 & 0.099291 & $0.092500-0.106320$ & 44 & 1.38160 & $1.32500-1.43950$ \\
\hline 20 & 0.113976 & $0.106320-0.121890$ & 45 & 1.53570 & $1.43950-1.63500$ \\
\hline 21 & 0.131230 & $0.121890-0.140910$ & 46 & 1.74330 & $1.63500-1.85540$ \\
\hline 22 & 0.152483 & $0.140910-0.164510$ & 47 & 1.92930 & $1.85540-2.00500$ \\
\hline 23 & 0.179012 & $0.164510-0.194120$ & 48 & 2.18960 & $2.00500-2.38240$ \\
\hline 24 & 0.212405 & $0.194128-0.231510$ & 49 & 2.54350 & $2.38240-2.71000$ \\
\hline 25 & 0.254637 & $0.231510-0.278860$ & 50 & 2.88180 & $2.71000-3.05900$ \\
\hline
\end{tabular}




\section{RECOMMENDATIONS FOR GENERATION OF THERMAL GROUP CONSTANTS}

\section{SPECTRUM}

Thermal group constants should be generated with a unit spectrum (weight 1.0 at all energies) so that they are applicable to all thermal spectrum reactors.

\section{RESONANCE CROSS SECTIONS}

A number of fissile and fertile isotopes have resonances below the $3.15 \mathrm{eV}$ maximum energy of the 460 group standard thermal energy group structure. Doppler broadening and self-shielding effects are important for many of these isotopes, although the self-shielding effects are highly dependent on geometry and spectrum.

Resonances lying below $3.15 \mathrm{eV}$ and any tails of resonances that extend below this energy should be included in thermal group constants at infinite dilution with Doppler broadening. A sufficient number of temperatures must be included in the group constants to assure accurate interpolation at intermediate temperatures.

\section{SCATTER ING MATRICES}

$A$ basic set of 30 group thermal scattering matrices prepared from ENDF/B $S(\alpha, \beta)$ data is recommended for thermal data testing. These scattering matrices are available from the Argonne Code Center ${ }^{11}$ (ACC Abstract 543) and consist of thermal scattering matrices up to $P_{5}$ Legendre order for the materials shown in Table III. These thermal scattering matrices are documented in Reference 12.

Included in the same package from the Argonne Code Center (ACC Abstract 543) are 50 group scattering matrices for the materials shown in Table IV. 
TABLE III

30 Group THERMOS Scattering Matrices Included in ACC Abstract 543

Material (ENDF/B MAT Number)

Temperatures, ${ }^{\circ} \mathrm{K}$

$\mathrm{H}_{2} \mathrm{O}(1002)$

$\mathrm{D}_{2} \mathrm{O}(1004)$

$350,400,450,500,600,700,800$

Graphite (1065)

$\mathrm{Zr}$ bound in $\mathrm{ZrH}_{\mathrm{X}}$ (1096)

$\mathrm{H}$ bound in $\mathrm{ZrH}_{\mathrm{X}}$ (1097)

$\left(\mathrm{C}_{2} \mathrm{H}_{4}\right)_{\mathrm{x}}$ Polyethylene (1011)

Be (1064)

BeO (1099)

$\mathrm{C}_{6} \mathrm{H}_{6}$ Benzene (1095)

$\mathrm{UO}_{2}$ (1098)

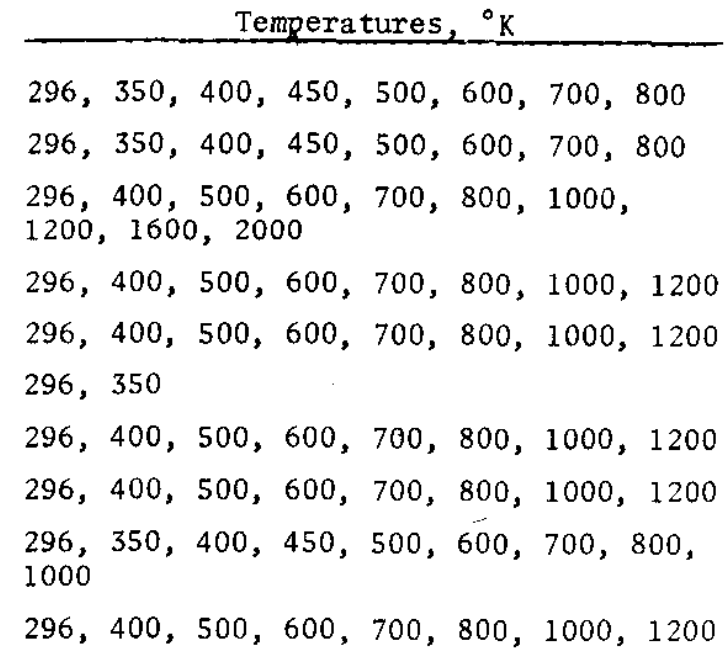

TABLE IV

50 Group Thermal Scattering Matrices Included in ACC Abstract 543

Material (ENDF/B MAT)

$\mathrm{H}_{2} \mathrm{O}$ (1002)

$\mathrm{H}$ bound in $\mathrm{H}_{2} \mathrm{O}$ (1002)

Free Gas Oxygen (1134)
Temperatures, ${ }^{\circ} \mathrm{K}$

$296,350,400,450,500,600,700,800$

$296,350,400,450,500,600,700,800$

$296,350,400,450,500,600,700,800$

\section{OUTPUT FORMAT}

The Codes and Formats Subcommittee of CSEWG has adopted the formats ${ }^{13}$ specified by the Committee on Computer Code Coordination for all standard group constants generated from ENDF/B data. 


\section{COMPUTER CODES FOR GENERATION OF THERMAL GROUP CONSTANTS}

Several computer codes exist that are capable of producing the required standard thermal group constants. Some of these codes may require modification to handle 460 energy groups. Table $V$ shows some of the available codes and the differences in their methods of generation of group constants from the above recommendations.

TABLE $V$

Thermal Region Cross Section Codes Interfaced to ENDF/B Data

Code Name

FLANGE II (Version 71-1) 14 ETOT $^{15}$
Comments

Can handle only 200 energy groups

Does not allow Doppler broadening of resonance cross sections

Cannot process $S(\alpha, \beta)$ data

The following two codes may be used together to generate group constants :

RESEND $^{26}$

Treats only room temperature data

INTEND $^{17}$ Does not handle thermal scattering matrices

Table VI shows the group equivalences between the standard thermal energy group structure and energy group structures for the thermal spectrum codes used to generate the compendium structures. 
TABLE VI

Equivalences Between Group Structures

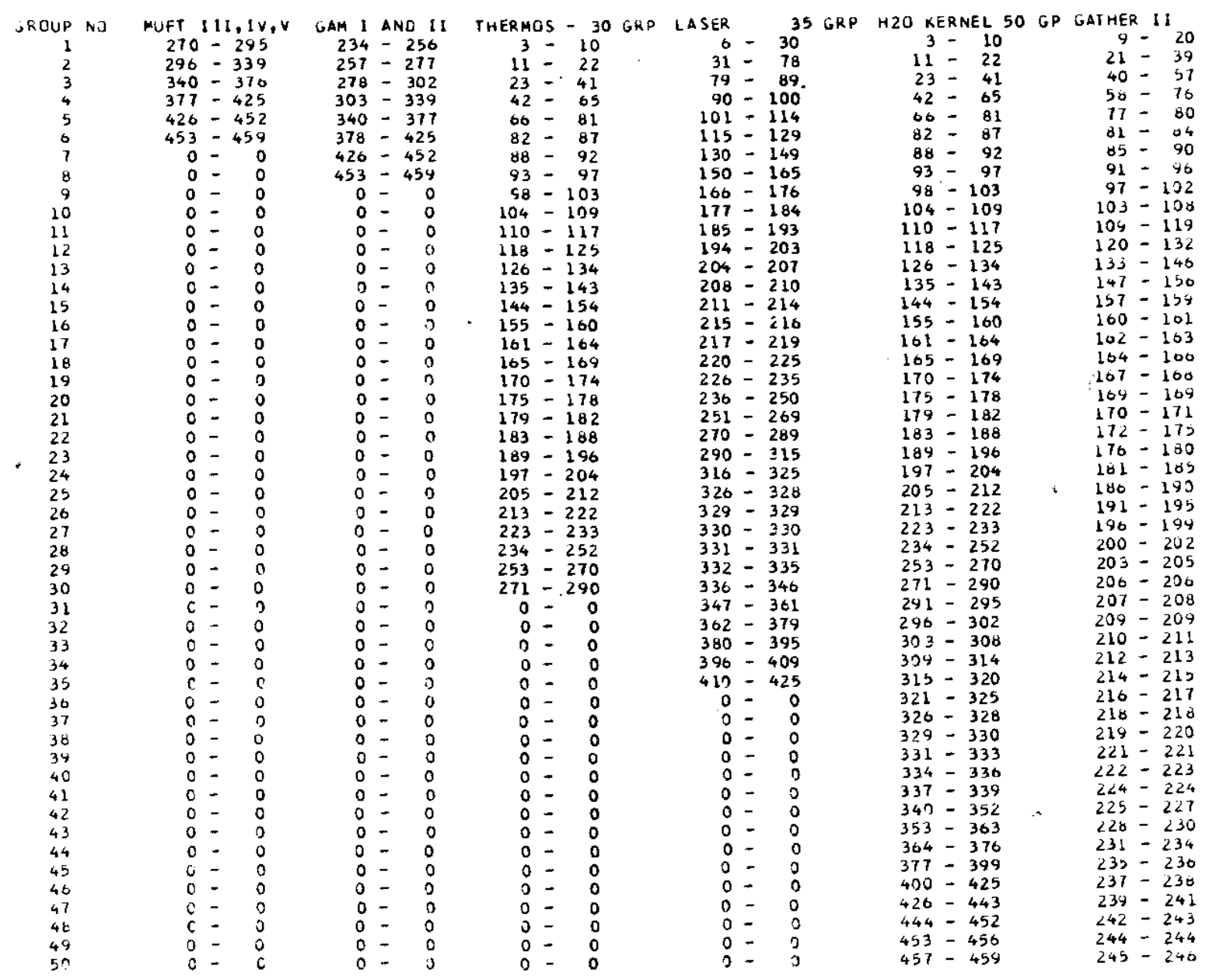




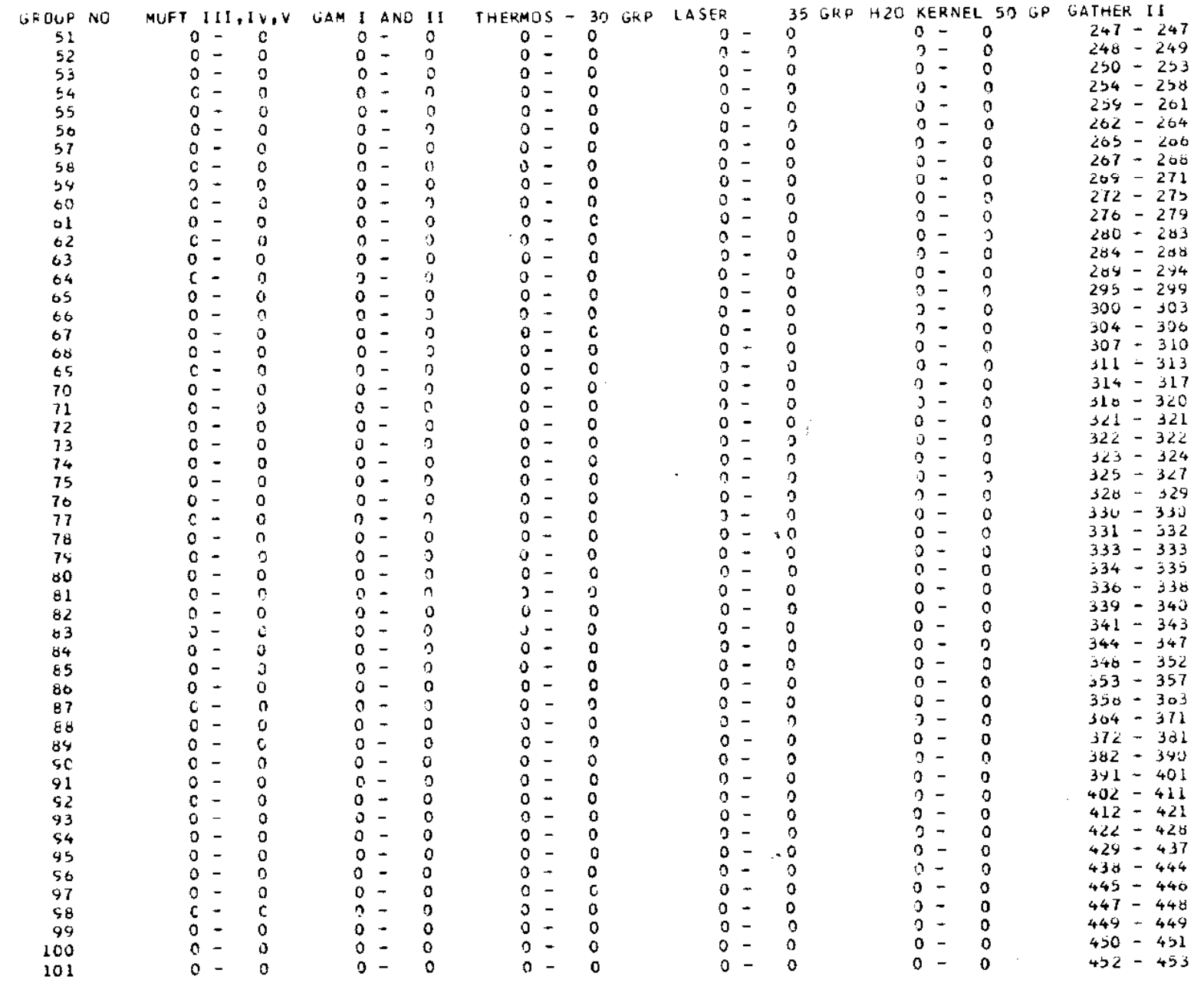




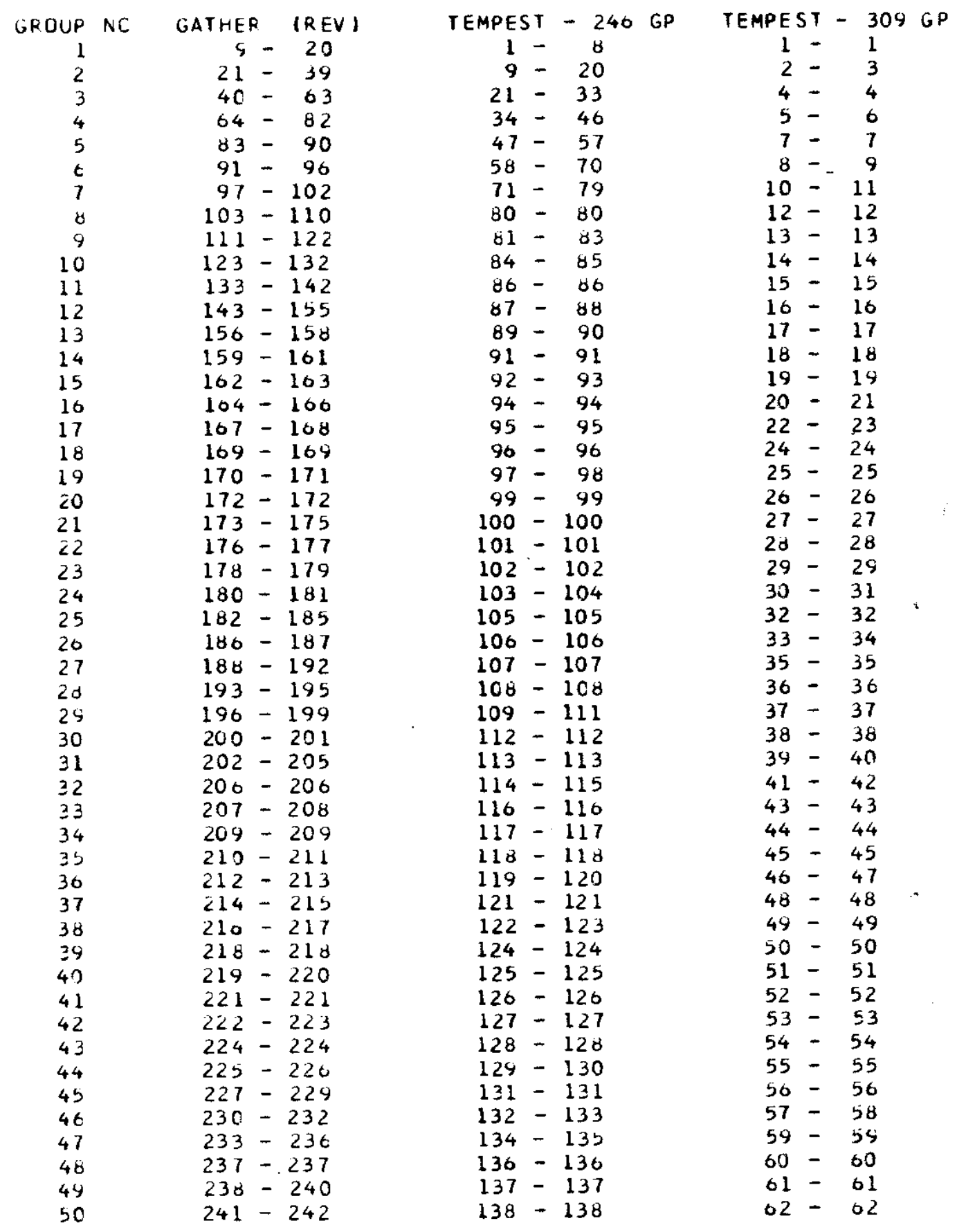




\begin{tabular}{|c|c|c|c|}
\hline GROUP & NC & GATHEF & (REV) \\
\hline 51 & & 243 & $-24 t$ \\
\hline 52 & & 247 & -248 \\
\hline 53 & & 249 & -251 \\
\hline 54 & & 252 & -254 \\
\hline 55 & & 255 & -259 \\
\hline 56 & & 260 & -262 \\
\hline 57 & & 263 & -265 \\
\hline 58 & & 260 & -268 \\
\hline 59 & & 269 & -271 \\
\hline 60 & & 272 & -274 \\
\hline 01 & & 275 & -278 \\
\hline$t 2$ & & 279 & -282 \\
\hline 63 & & 283 & -286 \\
\hline 64 & & 287 & -292 \\
\hline 65 & & 293 & -296 \\
\hline 66 & & 297 & -301 \\
\hline 67 & & 302 & -308 \\
\hline 68 & & $30 s$ & -313 \\
\hline 69 & & 314 & -319 \\
\hline 70 & & 320 & -322 \\
\hline 71 & & 323 & -323 \\
\hline 72 & & 324 & -326 \\
\hline 73 & & 327 & -327 \\
\hline 74 & & 328 & -328 \\
\hline 75 & & 329 & -329 \\
\hline 70 & & 330 & -330 \\
\hline 77 & & 331 & -333 \\
\hline 78 & & 334 & -334 \\
\hline 75 & & 335 & -337 \\
\hline 80 & & 338 & -338 \\
\hline 81 & & 339 & -341 \\
\hline 82 & & 342 & -344 \\
\hline 83 & & 345 & -347 \\
\hline 84 & & 348 & -351 \\
\hline 85 & & 352 & -357 \\
\hline 86 & & 358 & -303 \\
\hline 87 & & 364 & -371 \\
\hline 88 & & 372 & -379 \\
\hline 89 & & $38 \mathrm{C}$ & -390 \\
\hline 90 & & 391 & -401 \\
\hline 91 & & 402 & -418 \\
\hline 92 & & 419 & -431 \\
\hline 93 & & 432 & -445 \\
\hline$\$ 4$ & & 446 & -447 \\
\hline 95 & & 448 & -450 \\
\hline St & & 451 & -454 \\
\hline 97 & & 455 & -455 \\
\hline 58 & & 450 & -456 \\
\hline 99 & & 457 & -457 \\
\hline 100 & & 458 & $-45 \varepsilon$ \\
\hline
\end{tabular}

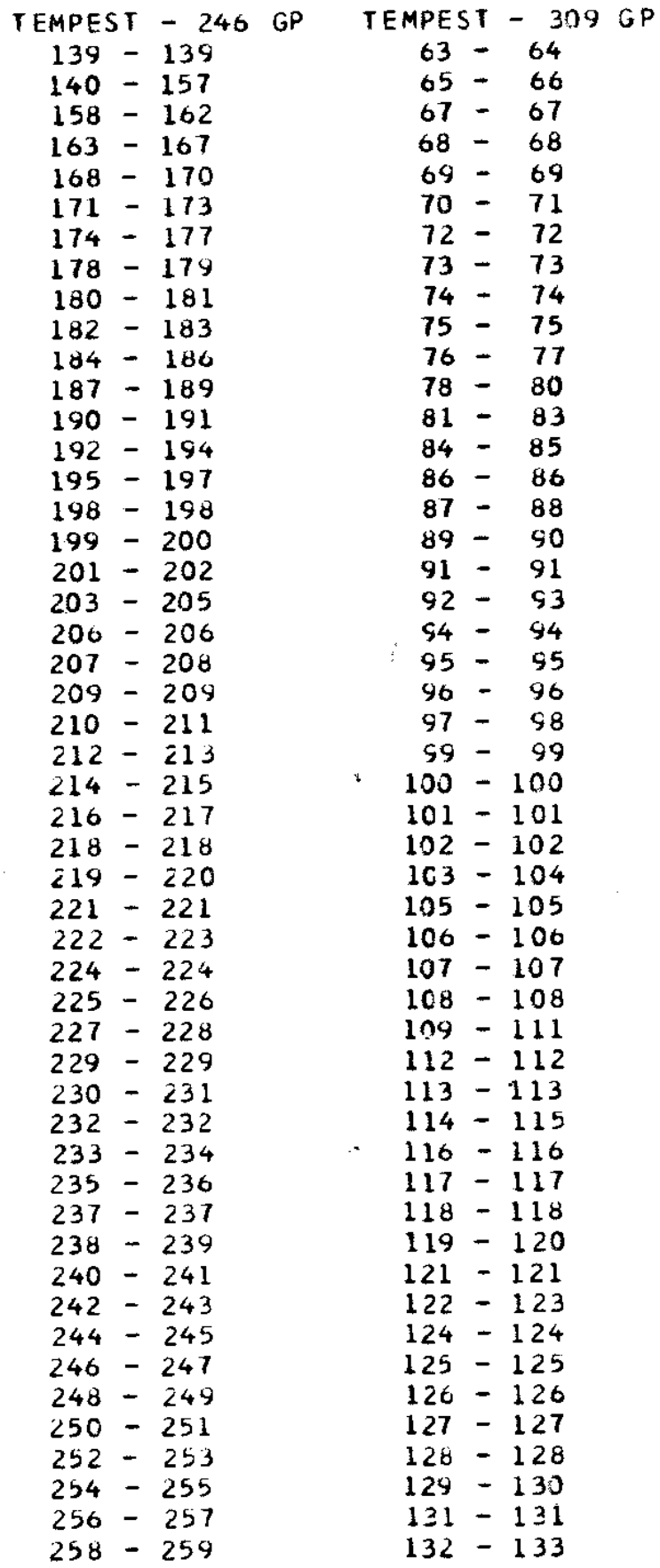




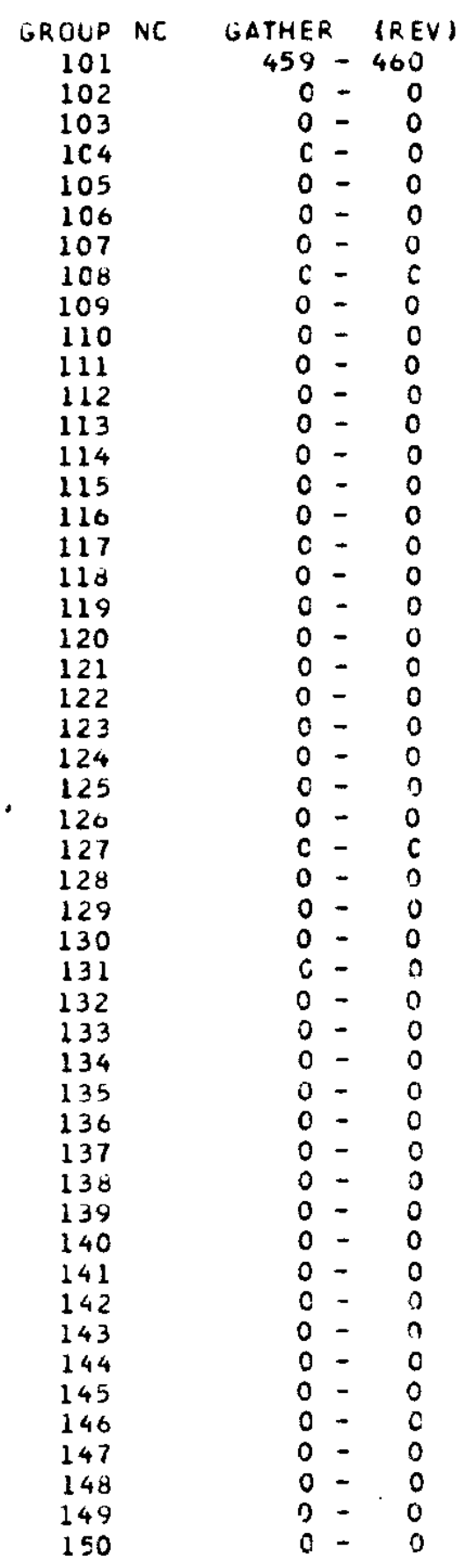

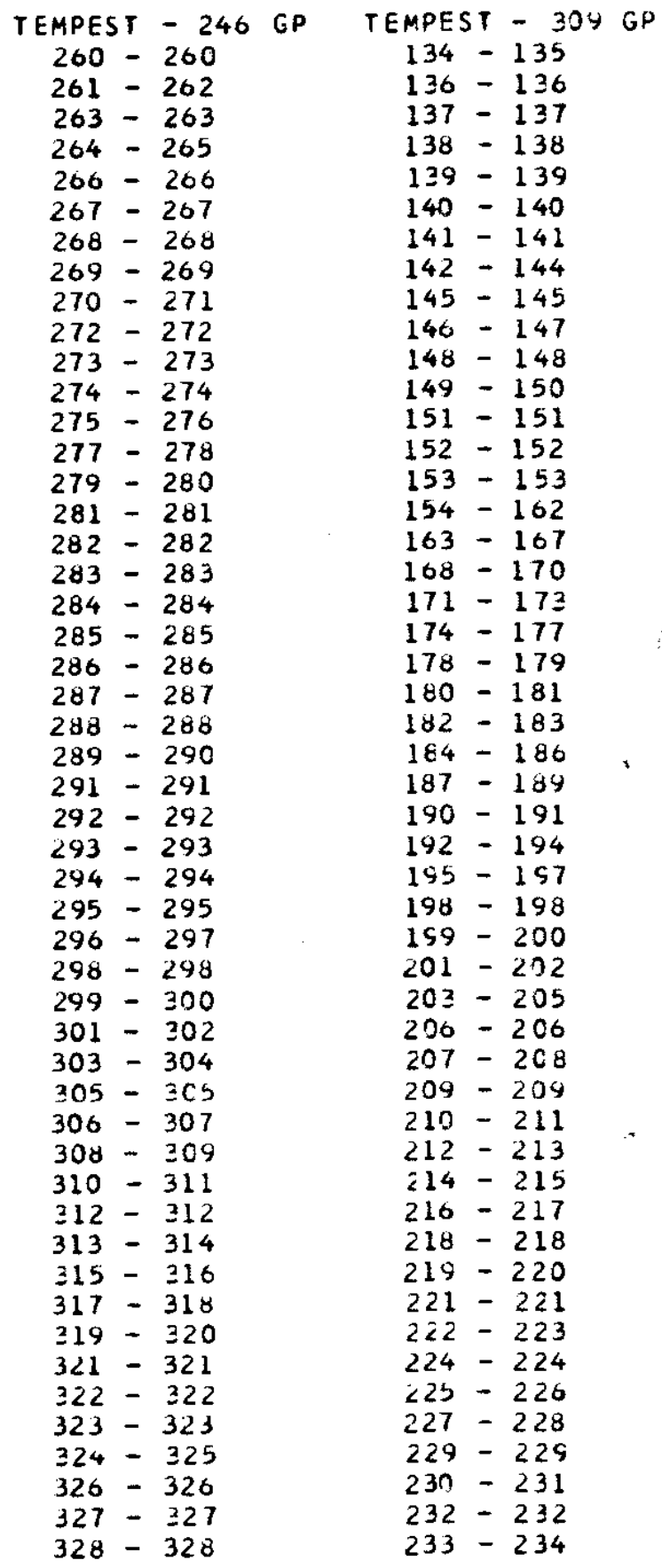




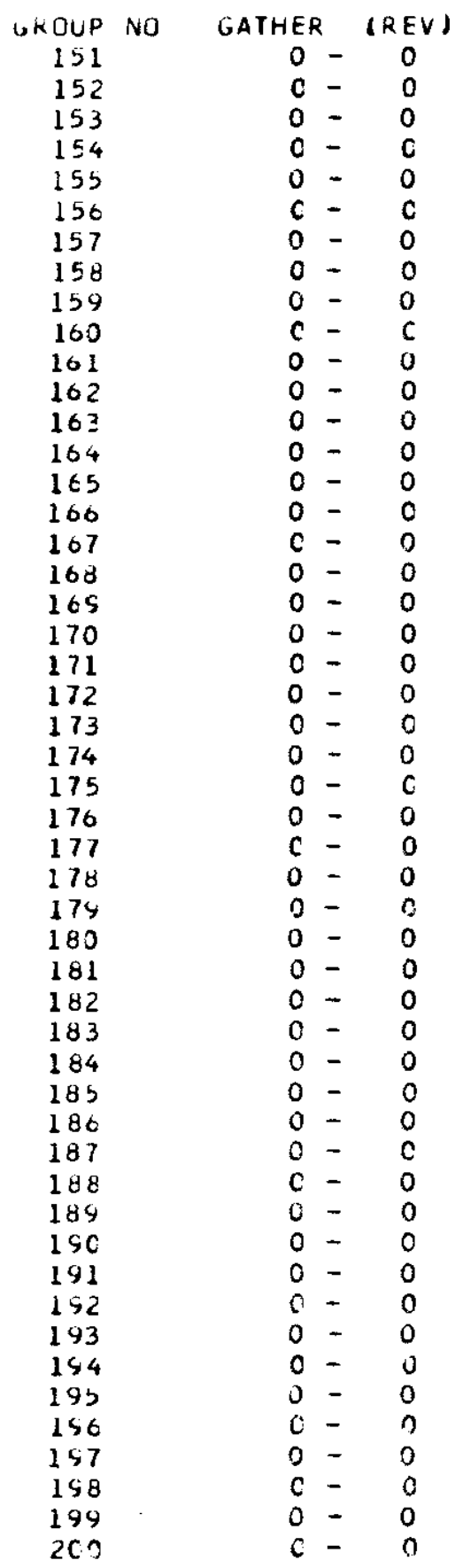

\begin{tabular}{|c|c|c|c|}
\hline EMPES & $T-246 G P$ & TEMPES & $\mathbf{T}-309 \mathrm{GP}$ \\
\hline 329 & -329 & 235 & -236 \\
\hline 330 & -330 & 237 & -237 \\
\hline 331 & -332 & 238 & -239 \\
\hline 333 & -333 & 240 & -241 \\
\hline 334 & -334 & 242 & -243 \\
\hline 335 & -336 & 244 & -245 \\
\hline 337 & -337 & 246 & -247 \\
\hline 338 & -339 & 248 & -249 \\
\hline 340 & -342 & 250 & -251 \\
\hline 343 & -344 & 252 & -253 \\
\hline 345 & -345 & 254 & -255 \\
\hline 346 & -346 & 256 & -257 \\
\hline 347 & -347 & 258 & -259 \\
\hline 348 & -348 & 260 & -260 \\
\hline 349 & -349 & 261 & -262 \\
\hline 350 & $-\quad 350$ & 263 & -263 \\
\hline 351 & -351 & 204 & -265 \\
\hline 352 & $-\quad 252$ & 266 & -266 \\
\hline 353 & -353 & 267 & -267 \\
\hline 354 & $-\quad 354$ & 208 & -268 \\
\hline 355 & -355 & 269 & -269 \\
\hline 356 & -356 & 270 & -271 \\
\hline 357 & -357 & 272 & -272 \\
\hline 358 & -358 & \& 273 & -273 \\
\hline 359 & -359 & 274 & -274 \\
\hline 360 & -360 & 275 & -276 \\
\hline 361 & -302 & 277 & -278 \\
\hline 363 & -363 & 279 & -280 \\
\hline 364 & -304 & 281 & -281 \\
\hline 305 & -365 & 282 & -282 \\
\hline 366 & -366 & 283 & -283 \\
\hline 367 & -367 & 284 & -284 \\
\hline 368 & -368 & 285 & -285 \\
\hline 369 & -369 & 286 & -286 \\
\hline 370 & -270 & 287 & -287 \\
\hline 371 & -372 & .288 & -288 \\
\hline 373 & -373 & 289 & -290 \\
\hline 374 & -374 & 291 & -291 \\
\hline 375 & $-\quad 375$ & 292 & -292 \\
\hline 370 & -377 & 293 & -293 \\
\hline 378 & $-\quad 378$ & 254 & -294 \\
\hline 379 & -380 & 295 & -295 \\
\hline 381 & -382 & 296 & -297 \\
\hline 383 & -383 & 298 & -298 \\
\hline 384 & -384 & 299 & -300 \\
\hline 385 & -385 & 301 & -302 \\
\hline 386 & -386 & 303 & -304 \\
\hline 387 & $-\quad 287$ & 305 & -305 \\
\hline 388 & -388 & 396 & -307 \\
\hline 389 & $-\quad 289$ & 308 & -309 \\
\hline
\end{tabular}




\begin{tabular}{|c|c|c|c|c|}
\hline GROLP & NO & GATHER & & (REV) \\
\hline 201 & & 0 & - & 0 \\
\hline 202 & & 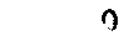 & - & 0 \\
\hline 203 & & 0 & - & 0 \\
\hline 204 & & $c$ & - & $c$ \\
\hline $20 b$ & & $n$ & - & 0 \\
\hline $20 t$ & & 0 & - & 0 \\
\hline 207 & & 0 & - & 0 \\
\hline 208 & & 2 & - & 0 \\
\hline 209 & & 0 & - & 0 \\
\hline 210 & & 0 & - & 0 \\
\hline 211 & & 0 & - & 0 \\
\hline 212 & & 0 & - & 0 \\
\hline 213 & & $c$ & - & 0 \\
\hline 214 & & 0 & - & 0 \\
\hline 215 & & 0 & - & 0 \\
\hline $21 t$ & & 0 & - & 0 \\
\hline 217 & & 0 & - & 0 \\
\hline 218 & & 0 & - & 0 \\
\hline 219 & & 0 & - & 0 \\
\hline 220 & & 0 & - & 0 \\
\hline $2<1$ & & c & - & 0 \\
\hline$\angle 22$ & & 0 & - & 0 \\
\hline 223 & & $C$ & - & c \\
\hline 224 & & 0 & - & 0 \\
\hline 225 & & 3 & - & 0 \\
\hline 220 & & 0 & - & 0 \\
\hline 227 & & $\mathrm{C}$ & - & C \\
\hline 228 & & 0 & - & 0 \\
\hline 224 & & 0 & - & 0 \\
\hline 230 & & 0 & - & 0 \\
\hline 231 & & 0 & - & 0 \\
\hline 232 & & c & - & 0 \\
\hline 233 & & 0 & - & 0 \\
\hline 234 & & 0 & - & 0 \\
\hline 235 & & 0 & - & 0 \\
\hline $23 t$ & & 0 & - & 0 \\
\hline 237 & & 0 & - & 0 \\
\hline 238 & & 0 & - & $c$ \\
\hline 234 & & 0 & - & 0 \\
\hline 240 & & 0 & - & 0 \\
\hline 241 & & 0 & - & 0 \\
\hline 242 & & 0 & - & 0 \\
\hline 243 & & 0 & - & 0 \\
\hline 244 & & 0 & - & 0 \\
\hline 245 & & 0 & - & 0 \\
\hline 246 & & 0 & - & 0 \\
\hline 247 & & 0 & - & ? \\
\hline 248 & & $c$ & - & 0 \\
\hline 249 & & 0 & - & 0 \\
\hline 250 & & 0 & - & 0 \\
\hline
\end{tabular}






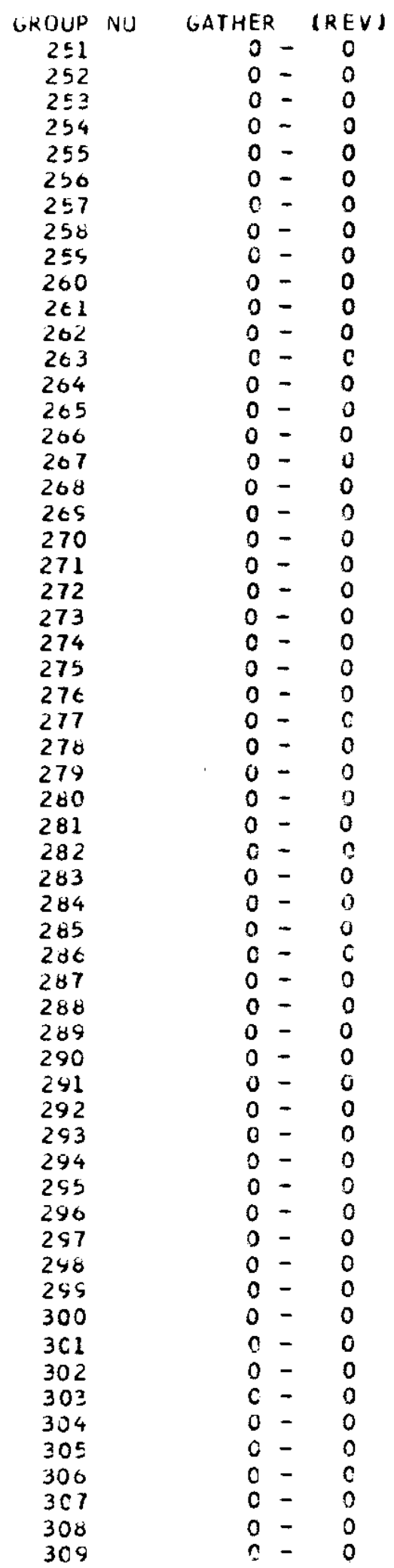

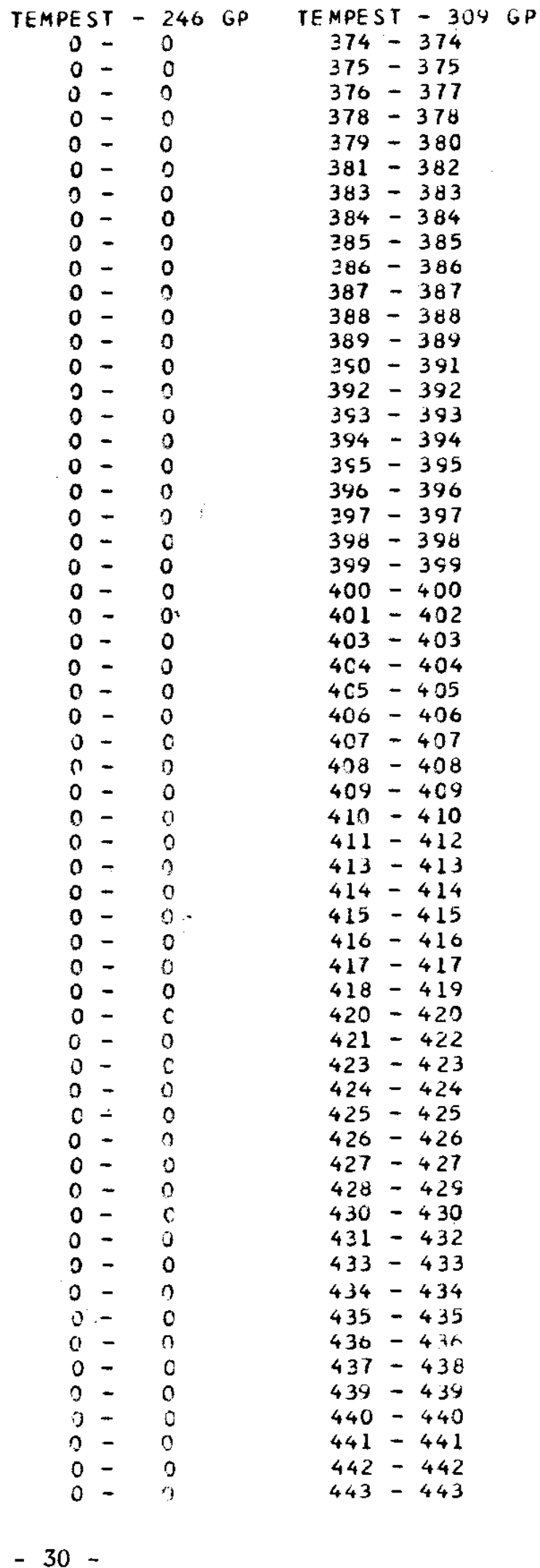




\section{ACKNOWLEDGMENTS}

Significant contributions were made to this work by a number of persons in the reactor physics community. The author would like to acknowledge the contributions of the following:

Don Mathews, Gulf General Atomic

Odelli Ozer, Brookhaven National Laboratory

Mahlen Raymond, Westinghouse Nuclear Energy Systems

Jud Hardy, Bettis Atomic Power Laboratory

Cecil Lubitz, Knolls Atomic Power Laboratory

Bob Dannels, Westinghouse Nuclear Energy Systems

W. Wittkopf, Babcock and Wilcox

H. W. Graves, Jr., University of Michigan

Peter Aline, General Electric Co. - APED

J. D. Jenkins, Oak Ridge National Laboratory

Duane Thompson, Battelle-Northwest Laboratory 


\section{REFERENCES}

1. C. R. Weisbin and R. J. LaBauve. Specification of a Generally Useful Multigroup Structure for Neutron Transport. USAEC Report LA-5277-MS, Los Alamos Scientific Laboratory, Los Alamos, N. M. (1973).

2. H. C. Honeck. THERMOS - A Thermalization Transport Theory Code for Reactor Lattice Calculations. USAEC Report BNL-5826, Brookhaven National Laboratory, Upton, N. Y. (1961).

3. C. G. Poncelet. LASER - A Depletion Program for Lattice Calculations Based on MUFT and THERMOS. USAEC Report WCAP-6073, Westinghouse Electric Co., Pittșburgh, Pa. (1966).

4. H. J. Amster and J. B. Callaghan. KATE-1 - A Program for Caloulating Wigner-Wilkins and Maswellian Averaged Thermal Constants on the Philco-2000. USAEC Report WAPD-TM-232, Westinghouse Electric Co., Pittsburgh, Pa. (1960).

5. E. L. Slaggie. Thermal Benchmark Calculations for WaterModerated Uranium- and Plutonium-Fueled Systems. USAEC Report GA-10337, Gulf Radiation Technology Co., San Diego, Calif. (1970).

6. R. H. Shudde. Preparation of $\sqrt{E} \sigma$ Cross Sections for the TEMPEST Decimal Library. AMTD-115, Westinghouse Electric Co., Pittsburgh, Pa. (1961).

7. H. Amster and R. Suarez. The Calculation of Thermal Constants Averaged Over a Wigner-Wilkins Flux Spectrum: Description of the SOFOCATE COde. USAEC Report WAPD-TM-39, Westinghouse Atomic Power Division, Pittsburgh, Pa. (1957).

8. R. F. Barry. LEOPARD - A Spectrum-Dependent Non-Spatial Depletion Code for the IBM-7094. USAEC Report WCAP-3269-26, Westinghouse Atomic Power Division, Pittsburgh, Pa. (1963).

9. H. Bah1, E. Gelbard, and G. Ryan. MUFT-4 - Fast Spectrum Code for the IBM-704. USAEC Report WAPD-TM-72, Westinghouse Atomic Power Division, Pittsburgh, Pa. (1957).

10. G. D. Joanou and J. S. Dudek. GAM-II: $A B_{3}$ Code for the Calculation of Fast-Neutron Spectra and Associated Multigroup Constants. USAEC Report GA-4265, General Atomic Div., General Dynamics Corp., San Diego, Calif. (1963). 
11. M. K. Butler, et al. Argonne Code Center: Compization of Progrom Abstracts. USAEC Report ANL-7411, Supplement 7, Argonne National Laboratory, Argonne, I11. (1973).

12. F. J. McCrosson, D. R. Finch, and E. C. Olson. Testing of ENDF/B - THERMOS CrOSs Sections for $\mathrm{H}_{2} \mathrm{O}, \mathrm{D}_{2} \mathrm{O}, \mathrm{C}, \mathrm{ZrH}_{2}$, $\left(\mathrm{C}_{2} \mathrm{H}_{4}\right)_{X}, \mathrm{Be}, \mathrm{BeO}, \mathrm{C}_{6} \mathrm{H}_{6}$, and $\mathrm{UO}_{2}$. USAEC Report DP-1276 (ENDF-158), E. I. du Pont de Nemours and Co., Savannah River Laboratory, Aiken, S. C. (1971).

13. B. M. Carmichae1. D. A. Meneley, and D. R. Vondy. Report of the Subcommittee on Standard Interface Files. USAEC Report LA-5324-MS, Los Alamos Scientific Laboratory, Los Alamos, N. M. (1973).

14. H. C. Honeck and D. R. Finch. FLANGE II (Version 71-1) - A Code to Process Thermal Neutron Data from an ENDF/B Tape. USAEC Report DP-1278 (ENDF-152), E. I. du Pont de Nemours and Co., Savannah River Laboratory, Aiken, S. C. (1971).

15. C. L. Beard and R. A. Danne1s. ETOT - A FORTRAN IV Progrom to Process Data from an ENDE/B Tape to Thermal Library Format. USAEC Report WCAP-7363 (ENDF-146), Westinghouse Nuclear Energy Systems, Pittsburgh, Pa. (1971).

16. 0. Ozer. RESEND - A Progrom to Pre-Process ENDF/B Materials with Resonance Files into a Pointwise Form. USAEC Report BNL-17134, Brookhaven National Laboratory, Upton, N. Y. (1972).

17. O. Ozer. INTEND - A General Purpose, Arbitrary Spectrum Integration Progrom for Pointwise ENDF/B Files. Private.. Communication (1973). 